\title{
Comparison of long-term outcomes between endoscopic band ligation and endoscopic clipping for colonic diverticular hemorrhage
}

Authors

Institution
Kaoru Nakano, Naoki Ishii, Takashi Ikeya, Mai Ego, Yuto Shimamura, Koichi Takagi, Kenji Nakamura, Katsuyuki Fukuda, Yoshiyuki Fujita

Department of Gastroenterology, St. Luke’s International Hospital, Tokyo, Japan
Bibliography

DOI http://dx.doi.org/

10.1055/s-0034-1392510

Published online: 11.8.2015

Endoscopy International Open

2015; 03: E529-E533

(c) Georg Thieme Verlag KG

Stuttgart $\cdot$ New York

E-ISSN 2196-9736

\section{Corresponding author}

Naoki Ishii, MD

Department of

Gastroenterology

St. Luke's International Hospital

9-1 Akashi-cho

Chuo-ku

Tokyo, Japan 104-8560

Fax: +81-3-3544-0649

naoishi0328@gmail.com
Background and study aims: Long-term outcomes of endoscopic band ligation (EBL) for colonic diverticular hemorrhage have not been reported to date. The aim of this study was to determine the long-term outcomes of EBL and to retrospectively compare them with those of endoscopic clipping (EC) in the treatment of colonic diverticular hemorrhage.

Patients and methods: The study included patients with colonic diverticular hemorrhage who were treated with EBL or EC from January 2004 to November 2014 and followed up more than 1 year (61 patients in the EBL group and 39 patients in the EC group). Time-to-event analysis of rebleeding was performed with the Kaplan-Meier method. A follow-up colonoscopy was performed to confirm the disappearance of the banded diverticula in the EBL group.

\section{Introduction \\ $\nabla$}

Incidence of colonic diverticular hemorrhages is rising due to increasing comorbidities, such as hypertension and arteriosclerosis, and regular use of antiplatelet agents or nonsteroidal anti-inflammatory drugs [ $1-6]$. Colonic diverticular hemorrhage is the most common cause of lower gastrointestinal bleeding (LGIB) [7-9], and is seen to resolve spontaneously in $70 \%$ to $90 \%$ of cases [7, $10-12]$. However, endoscopic treatment, surgery, or transarterial embolization (TAE) may be required in cases with severe or continuous bleeding [12-16].

Colonoscopy is useful for diagnosis of colonic diverticular hemorrhage, and endoscopic treatments for hemostasis include epinephrine injection, contact thermal therapy, and endoscopic clipping (EC) [12,17-22]. Recently, endoscopic band ligation (EBL) has also been performed to achieve hemostasis [23-28]. We previously reported that EBL was a safe and effective endoscopic treatment for colonic diverticular hemor-
Results: Rebleeding occurred in 21 patients in the EBL group and in 26 patients in the EC group. The cumulative incidence of rebleeding at $1,12,24$, and 36 months after first treatments was $14 \%$, $23 \%, 26 \%$, and $41 \%$ in the EBL group and $38 \%$, $49 \%, 59 \%$, and $68 \%$ in the EC group, respectively. Time-to-event analysis revealed statistically significant data (Log-rank test, $P=0.0036$ ). Scar formation with fold convergence at the previously banded site was observed in 11 of 24 patients who underwent follow-up colonoscopy (46\%). However, late rebleeding (rebleeding more than 30 days after EBL) occurred in five of these 11 cases.

Conclusions: EBL was superior to EC in the treatment of colonic diverticular hemorrhage. However, the risk of rebleeding was not avoided even after the diverticula had been resolved using EBL.

rhage, and that resolution of the colonic diverticula may prevent late rebleeding (rebleeding more than 30 days after initial treatment) [12] from the banded diverticula [28]. However, there are a limited number of reported cases of colonic diverticular hemorrhage treated with $\mathrm{EBL}$, and the long-term outcomes of the procedure have not been elucidated to date. The aim of this study is to investigate the long-term outcomes of EBL and to compare them with those for EC in treatment of colonic diverticular hemorrhage.

\section{Patients and methods \\ $\nabla$}

Study population

This study is a retrospective review of all patients who underwent colonoscopy for diagnosis and treatment of acute LGIB from January 2004 to November 2014 at St. Luke's International Hospital in Tokyo. First-line treatment for colonic diverticular hemorrhage was EC from January 2004 to 
734 patients who received colonoscopy due to acute LGIB

Postpolypectomy bleeding, colorectal cancer, angiodysplasia, ischemic colitis, ileal diverticular bleeding and incidental diverticulosis were ruled out.

Definite or presumptive colonic diverticular hemorrhage $(n=379)$

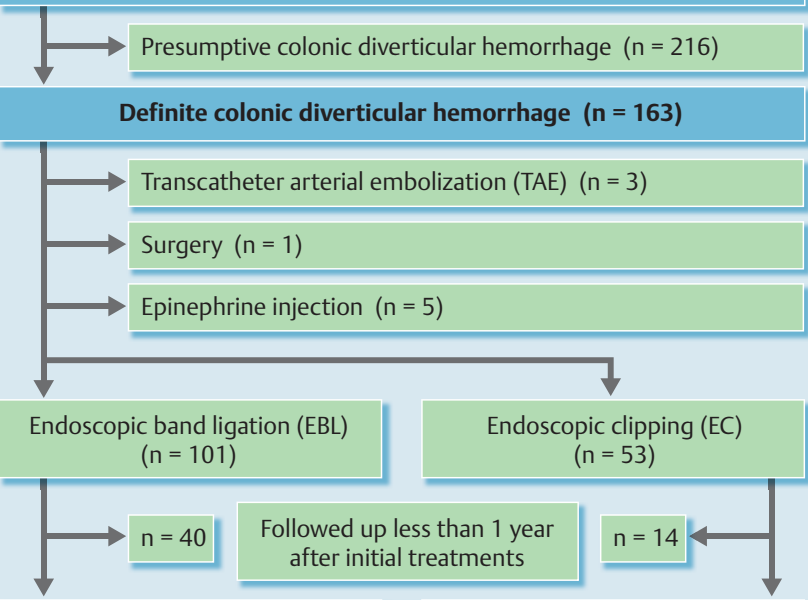

EBL group $(n=61)$

EC group $(n=39)$

Fig. 1 Flowchart of patients with colonic diverticular hemorrhage included in this study.

May 2009, and EBL from June 2009 to November 2014. A study flow diagram is presented in $\bullet$ Fig. 1.

Only cases of colonic diverticular hemorrhage were included, and all cases of bleeding (polypectomy bleeding, colorectal cancer, ischemic colitis, vascular ectasia, and ileal diverticular bleeding) were excluded. Presumptive diverticular hemorrhage was ruled out where the stigmata of recent hemorrhage (SRH) were absent in the colonoscopy, because it is essential for unequivocal identification of specific diverticula as the bleeding source. Sources were classified, using colonoscopy, into active bleeding (AB), non-bleeding visible vessel (NBVV), or adherent clots (AC) [12]. A total of 163 patients were diagnosed with definite colonic diverticular hemorrhage with SRH. Patients treated with TAE, surgery, or epinephrine injection therapy as first-line therapy were excluded. A total of 154 patients with definite diverticular hemorrhage were successfully treated with EBL or EC as first-line treatment. Patients who were followed up for less than 1 year after initial endoscopic treatments (EBL or EC) at St. Luke's International Hospital were excluded, resulting in a final sample of 61 patients in the EBL group and 39 patients in the EC group.

\section{Treatment methods}

Colonoscopy was performed after bowel preparation with polyethylene glycol, in both the EBL and the EC groups. However, patients with hemodynamic instability did not undergo bowel preparation. Endoscopic treatments were performed by welltrained endoscopists and a trainee under the supervision of experts.

The EBL method was performed as follows ( Fig.2) [25-28]: Once the diverticula were identified with SRH, they were marked with hemoclips and the endoscope was removed. It was then reinserted after a band-ligator device (MD-48710 EVL Device, Sumitomo Bakelite Co., Tokyo, Japan) was attached to its tip. When the endoscope head reached the target point, the bleeding diverticula were suctioned into the band-ligator cup. Thereafter, the $\mathrm{O}-$ band was released to ligate the inverted diverticula. Tattooing was not performed at the bleeding point. If EBL was not successful, other endoscopic treatments, such as endoscopic clipping or epinephrine injection therapy, were performed.

EC was performed as previously reported ( $\bullet$ Fig.3) [22], i.e., by direct placement of hemoclips (HX-600-090 L, HX-600-135, HX610-090 L, or HX-610-135; Olympus Medical Systems, Tokyo, Japan), if feasible, on the targeted vessel. When direct placement was considered difficult because of the dome location or massive hemorrhage, indirect placement (closing with multiple hemoclips in a zipper fashion) was performed.

\section{Follow-up after endoscopic treatments}

After endoscopic treatment, patients were followed up on an outpatient basis at St. Luke's International Hospital. Continuous massive hematochezia after endoscopic treatments was considered as rebleeding. In such situations, a repeat colonoscopy was performed to retreat the diverticula, or to rule out other bleeding lesions, such as colonic neoplasms or vascular ectasia. If required, additional treatments were administered based on the judgment of the attending gastroenterologists.

In the EBL group, follow-up colonoscopy was performed after the procedure to confirm the disappearance of the banded diverticula, except in patients with severe comorbid conditions such as cardiovascular disease. A convergence of folds at the site of the previously banded diverticula, seen on follow-up colonoscopy, was considered as the disappearance of the diverticula with SRH

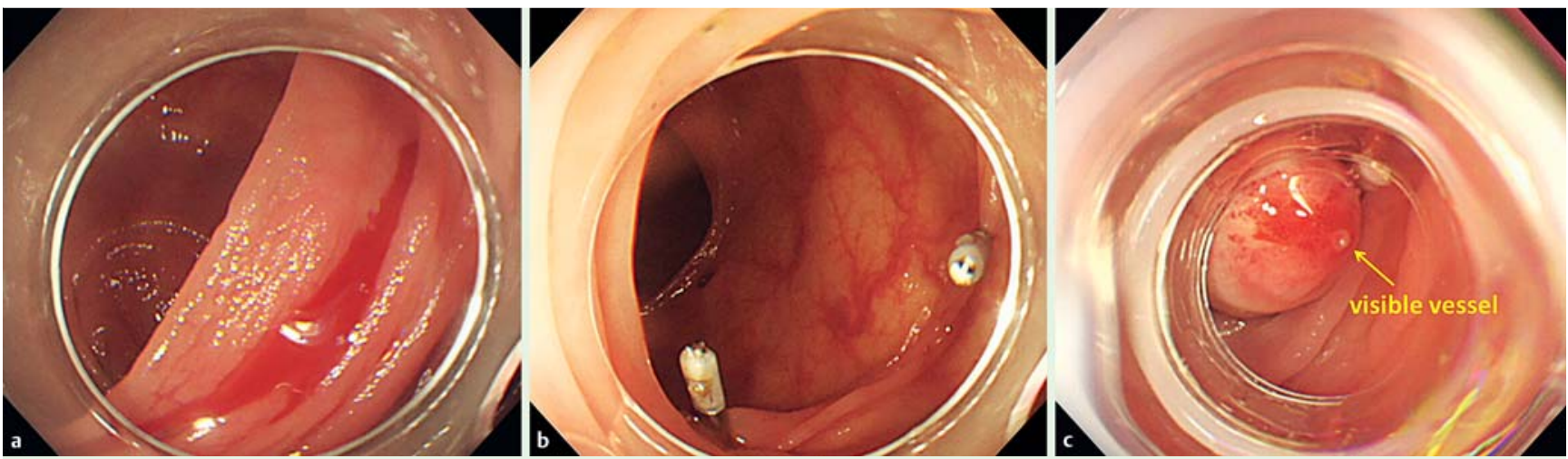

Fig. 2 a Endoscopic band ligation (EBL) for colonic diverticular hemorrhage. Endoscopic view of the colonic diverticulum with active bleeding. b Marking with hemoclips was performed near the diverticulum. $\mathbf{c}$ The elastic O-ring was released and a visible vessel was observed on the banded diverticulum. 

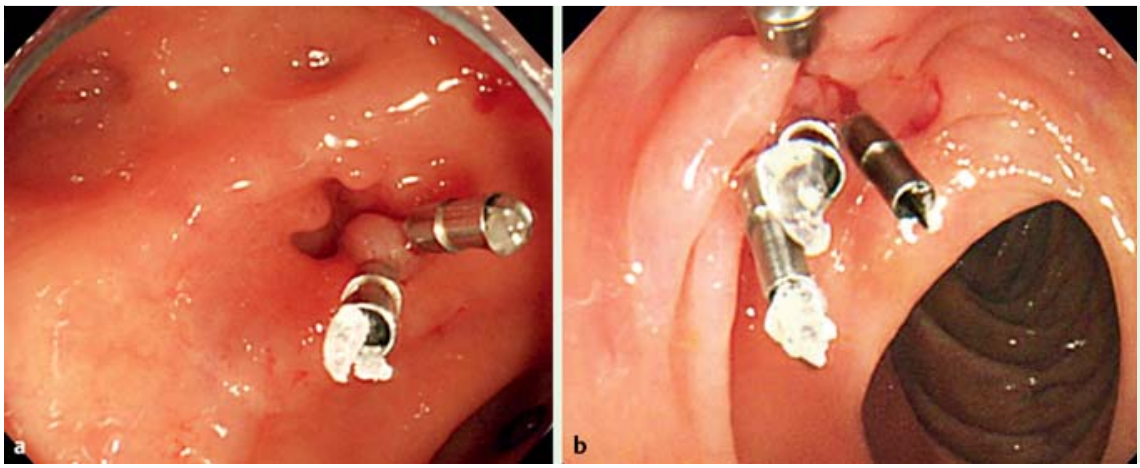

Fig. 3 a Endoscopic clipping (EC) for colonic diverticular hemorrhage. Direct placement of hemoclips on the targeted vessel was performed. $\mathbf{b}$ Indirect placement of hemoclips was performed in a zipper fashion.

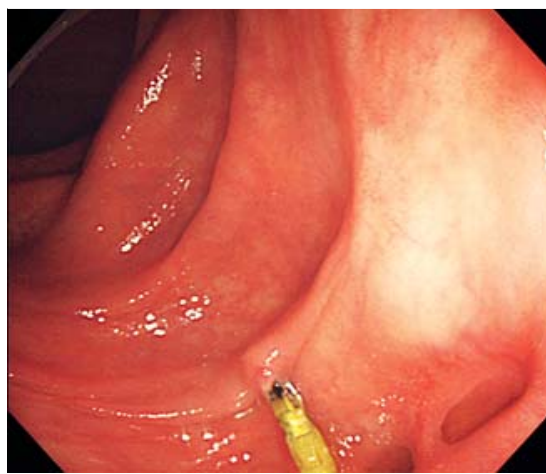

Fig. 4 Scar formation was observed at the previously banded site in a follow-up colonoscopy.

$(\bullet$ Fig.4) [28]. However, the presence or absence of the previously banded diverticula could not be confirmed in all cases because tattooing was not performed near the bleeding point. In such cases, we recorded the results as unknown. This study was approved by the ethics committee of our hospital, and written informed consent was obtained from all patients. The patients who were not followed up at St. Luke's International Hospital were contacted by telephone to confirm the presence or absence of massive hematochezia after endoscopic treatments.

\section{Statistical analysis}

Statistical analysis was performed using JMP version 9 (SAS Institute Inc., USA). Patients' ages and follow-up periods after initial treatment were reported as mean [standard deviation (SD)] and median (range), respectively. Student's t-test or a Mann-Whitney $U$-test were used for continuous variables, and the Fisher's exact test was used for categorical variables. A $P$ value less than 0.05 was considered statistically significant. Time-to-event analysis of rebleeding was analyzed using the Kaplan-Meier method.

\section{Results}

$\nabla$

\section{Characteristics of study groups}

The characteristics of patients with definite diverticular hemorrhage treated with EBL or EC are presented in - Table 1. A statistically significant difference was seen in hypertension comorbidity and median follow-up periods after initial treatments $(P<$ $0.001)$. No difference was seen, however, in the other parameters. Direct placement of hemoclips was performed in 10 out of 39 patients.

\section{Follow-up profile after initial treatments}

During the follow-up period, rebleeding occurred in 21 patients from the EBL group and 26 patients from the EC group. It was also seen in 19 of 29 patients treated using indirect placement of EC, and 7 of 10 patients treated with direct placement. Early rebleeding (rebleeding within 30 days after endoscopic treatments) [12] occurred in 12 patients in the indirect group and three patients in the direct placement group. The median number of days (range) before occurrence of rebleeding was 92 days (range; 0-934 days) and 874 days (range; $0-1780$ days) in the EBL and EC groups, respectively. Management of rebleeding in the EBL group was as follows: conservative therapy, $n=13$; $E B L$, $\mathrm{n}=6$; EC, $\mathrm{n}=1$; TAE, $\mathrm{n}=0$; and surgery, $\mathrm{n}=1$. Right hemicolectomy was performed in one ascending case, as per the patient's preference and not because of uncontrolled bleeding. Management of rebleeding in the EC group was as follows: conservative therapy, $\mathrm{n}=8$; EBL, $\mathrm{n}=2$; $E C, \mathrm{n}=12$; TAE, $\mathrm{n}=3$; and surgery, $\mathrm{n}=1$. TAE or surgery was performed because of uncontrolled bleeding in four cases in the EC group.

\begin{tabular}{|lllc|}
\hline & EBL $(\mathbf{n = 6 1 )}$ & EC (n=39) & P value \\
\hline Male, $\mathrm{n}(\%)$ & $44(72)$ & $29(74)$ & 0.81 \\
\hline Age, mean (SD) & $67(13)$ & $64(13)$ & 0.32 \\
\hline Antiplatelet agent, $\mathrm{n}(\%)$ & $18(30)$ & $13(33)$ & 0.69 \\
\hline NSAIDs, $\mathrm{n}(\%)$ & $4(7)$ & $3(8)$ & 0.83 \\
\hline Hypertension, $\mathrm{n}(\%)$ & $33(55)$ & $19(49)$ & $<0.001^{1}$ \\
\hline Right colon, $\mathrm{n}(\%)$ & $45(74)$ & $28(72)$ & 0.25 \\
\hline AB : NBVV+ AC, $\mathrm{n}(\%)$ & $25(41): 36(59)$ & $16(41): 23(59)$ & 1.00 \\
\hline Follow-up periods, median (range) (months) & $30(12-65)$ & $65(12-111)$ & $<0.001^{1}$ \\
\hline
\end{tabular}

Table 1 Characteristics of patients with definite diverticular hemorrhage treated with endoscopic band ligation (EBL) or endoscopic clipping (EC).

NSAIDs, non-steroidal anti-inflammatory drugs; $A B$, active bleeding; NBVV, non-bleeding visible vessel; AC, adherent clot. ${ }^{1} \mathrm{~A} P$ value less than 0.05 was considered statistically significant. 


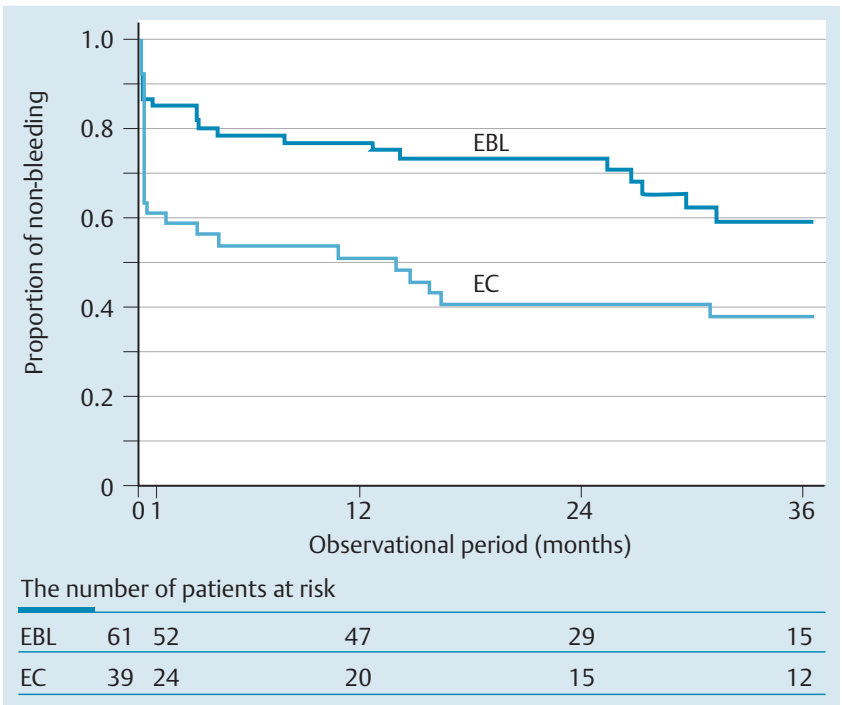

Fig. 5 Kaplan-Meier estimates of rebleeding after successful EBL or EC for colonic diverticular hemorrhage.

\section{Long-term outcomes of EBL or EC for diverticular hemorrhage}

The Kaplan-Meier test estimates of rebleeding in each group are presented in $\odot$ Fig. 5 . In the EBL group, the cumulative incidence of rebleeding at $1,12,24$, and 36 months were $14 \%, 23 \%, 26 \%$, and $41 \%$, respectively. In the EC group, the cumulative incidence of rebleeding at $1,12,24$, and 36 months were $38 \%, 49 \%, 59 \%$, and $68 \%$, respectively. The time-to-event analysis revealed statistically significant data (Log-rank test, $P=0.0036$ ). A follow-up colonoscopy was performed in 24 out of 61 EBL cases (39\%). Scar formation with fold convergence at the previously banded site, which was considered as complete resolution of the diverticula, was observed in 11 cases (46\%). However, late rebleeding occurred in 5 out of 11 cases.

\section{Discussion \\ $\nabla$}

Recently, endoscopic hemostatic methods such as epinephrine injection, contact thermal therapy, EC, and EBL [12,17-28] have become the most common treatments for colonic diverticular hemorrhage. In our retrospective cohort study, we investigated long-term follow-up results with EBL and EC for treatment of colonic diverticular hemorrhage.

The walls of the colon are thin, and the absence of a muscular layer in the colonic diverticula implies a risk of perforation during contact thermal therapy and/or epinephrine injection. Therefore, EC is sometimes preferred over contact coagulation therapy for treatment of colonic diverticular hemorrhage, because it provides immediate mechanical hemostasis and causes less injury to the colonic tissues [18-22]. However, direct placement of hemoclips on the targeted vessel is difficult, particularly in cases of dome location, massive hemorrhage, or small diverticular orifices $[20-22]$. Moreover, the colonic diverticular anatomy sometimes precludes indirect placement of hemoclips at the diverticula for occlusion of either the SRH or the underlying artery [29], and bleeding from right-sided diverticulosis (commonly seen in eastern countries) is more severe than that from left-sided diverticulosis [30]. High rates of rebleeding after EC were seen in the current study in which 29 patients (74\%) were treated using the in- direct method and 28 bleeding diverticula (72\%) were located in the right colon.

Recently, EBL has been performed to treat colonic diverticular hemorrhage with the band-ligator device that is widely used for esophageal varices [25-28]. In the current study, the rate of rebleeding was significantly lower in the EBL group than in the EC group. By occluding SRH and underlying arteries using EBL, higher hemostatic effects and lower rebleeding rates may be obtained, and late bleeding from the "same" diverticula could be prevented by resolution of the banded diverticula.

However, late rebleeding occurred in five cases where the followup colonoscopy showed resolution of the banded diverticula. These results imply that late rebleeding occurred from different diverticula, implying that it cannot be prevented even after resolution of the previously banded diverticula.

The main limitation of this study was the difference in the followup periods between the EBL and EC groups. In our institution, until May 2009, colonic diverticular hemorrhage had been treated mainly with EC; in June 2009, it was replaced by EBL. Therefore, rebleeding after initial treatments were compared between the groups using the Kaplan-Meier method.

In conclusion, although the current study was retrospective and the number of patients followed up long term was limited, EBL was considered superior to EC in treatment of colonic diverticular hemorrhage. However, the risk of rebleeding could not be avoided, even after resolution of the diverticula using EBL.

\section{Competing interests: None}

\section{References}

1 Foutch PG. Diverticular bleeding: are nonsteroidal anti-inflammatory drugs risk factors for hemorrhage and can colonoscopy predict outcome for patients? Am J Gastorenterol 1995; 90: 1779-1784

2 Aldoori WH, Giovannucci EL, Rimm EB et al. Use of acetaminophen and nonsteroidal anti-inflammatory drugs: a prospective study and the risk of symptomatic diverticular disease in men. Arch Fam Med 1998; 7: $255-260$

3 Yamada A, Sugimoto T, Kondo $S$ et al. Assessment of the risk factors for colonic diverticular hemorrhage. Dis Colon Rectum 2008; 51: 116 120

4 Strate LL, Liu YL, Huang ES et al. Use of aspirin or nonsteroidal anti-inflammatory drugs increases risk for diverticulitis and diverticular bleeding. Gastroenterology 2011; 140: 1427-1433

5 Niikura R, Nagata N, Akiyama J et al. Hypertension and concomitant arteriosclerotic diseases are risk factors for colonic diverticular bleeding: a case-control study. Int J Colorectal Dis 2012; 27: 1137-1143

6 Okamoto T, Watabe H, Yamada A et al. The association between arteriosclerosis related diseases and diverticular bleeding. Int J Colorectal Dis 2012; 27: $1161-1166$

7 Longstreth GF. Epidemiology and outcome of patients hospitalized with acute lower gastrointestinal hemorrhage: a population-based study. Am J Gastroenterol 1997; 92: 419-424

8 Chaudhry V, Hyser MJ, Gracias VH et al. Colonoscopy: the initial test for acute lower gastrointestinal bleeding. Am Surg 1998; 64: 723 - 728

9 Newman J, Fitzgerald JE, Gupta $S$ et al. Outcome predictors in acute surgical admissions for lower gastrointestinal bleeding. Colorectal Dis 2012; 14: 1020 - 1026

10 McGuire HH Jr. Bleeding colonic diverticula. A reappraisal of natural history and management. Ann Surg 1994; 220: 653-656

11 Poncet $G$, Heluwaert F, Voirin D et al. Natural history of acute colonic diverticular bleeding: a prospective study in 133 consecutive patients. Aliment Pharmacol Ther 2010; 32: 466-471

12 Jensen DM, Machicado GA, Jutabha $R$ et al. Urgent colonoscopy for the diagnosis and treatment of severe diverticular hemorrhage. N Engl J Med 2000: 342; 78-82

13 Suzman MS, Talmor M, Jennis R et al. Accurate localization and surgical management of active lower gastrointestinal hemorrhage with technetium-labeled erythrocyte scintigraphy. Ann Surg 1996; 224: 29-36 
14 Farrell JJ, Friedman LS. Gastrointestinal bleeding in the elderly. Gastroenterol Clin North Am 2001; 30: 377-407

15 Funaki B, Kostelic JK, Lorenz J et al. Superselective microcoli embolization of colonic hemorrhage. AJR Am J Roentgenol 2001; 177: 829-836

16 Tan KK, Nallathamby $V$, Wong $D$ et al. Can superselective embolization be definitive for colonic diverticular hemorrhage? An institution's experience over 9 years J Gastrointest Surg 2010; 14: 112 - 118

17 Bloomfeld RS, Rockey DC, Shetzline MA. Endoscopic therapy of acute diverticular hemorrhage. Am J Gastroenterol 2001; 96: 2367-2372

18 Hokama A, Uehara T, Nakayoshi T et al. Utility of endoscopic hemoclipping for colonic diverticular bleeding. Am J Gastroenterol 1997; 92: $543-546$

19 Simpson PW, Nguyen MH, Lim JK et al. Use of endoclips in the treatment of massive colonic diverticular bleeding. Gastrointest Endosc 2004; 59: $433-437$

20 Yen EF, Ladabaum U, Muthusamy VR et al. Colonoscopic treatment of acute diverticular hemorrhage using endoclips. Dig Dis Sci 2008; 53: $2480-2485$

21 Kaltenbach T, Watson R, Shah J et al. Colonoscopy with clipping is useful in the diagnosis and treatment of diverticular bleeding. Clin Gastroenterol Hepatol 2012; 10: 131-137

22 Ishii $N$, Hirata $N$, Omata $F$ et al. Location in the ascending colon is a predictor of refractory colonic diverticular hemorrhage after endoscopic clipping. Gastrointest Endosc 2012; 76: 1175-1181
23 Witte JT. Band ligation for colonic bleeding: modification of multiband ligating devices for use with a colonoscope. Gastrointest Endosc 2000; 52: $762-765$

24 Farrell JJ, Graeme-Cook F, Kelsey PB. Treatment of bleeding colonic diverticula by endoscopic band ligation: an in-vivo and ex-vivo pilot study. Endoscopy 2003; 35: 823-829

25 Ishii $N$, Itoh $T$, Uemura $M$ et al. Endoscopic band ligation with a waterjet scope for the treatment of colonic diverticular hemorrhage. Dig Endosc 2010; 22: $232-235$

26 Ishii $N$, Uemura $M$, Itoh $T$ et al. Endoscopic band ligation for the treatment of bleeding colonic and ileal diverticula. Endoscopy 2010; 42: $82-83$

27 Setoyama T, Ishii N, Fujita Y et al. Endoscopic band ligation (EBL) is superior to endoscopic clipping for the treatment of colonic diverticular hemorrhage. Surg Endosc 2011; 25: 3574 -3578

28 Ishii N, Setoyama T, Deshpande GA et al. Endoscopic band ligation (EBL) for colonic diverticular hemorrhage. Gastrointest Endosc 2012; 75 : $382-387$

29 Meyers MA, Alonso DR, Gray GF et al. Pathogenesis of bleeding colonic diverticulosis. Gastroenterology 1976; 71: 577-583

30 Wong SK, Ho YH, Leong AP et al. Clinical behavior of complicated rightsided and left-sided diverticulosis. Dis Colon Rectum 1997; 40: $344-$ 348 